\title{
Transvenous Target Embolization for a Report Small Sized, Non-traumatic Direct Carotid-cavernous Fistula using a Single Coil: Technical Case Report
}

Nobuhide Hayashi, Hideo Okada, Nagatsuki Tomura, Takahiro Sasaki, and Toshikazu Kuwata

Purpose: We report on a patient who underwent transvenous target embolization of a small, direct carotid-cavernous fistula (CCF).

Case: A 74-year-old female without a history of trauma was referred to our department. Tinnitus and ocular symptoms initially occurred as symptoms of a right, direct CCF. Anterograde blood flow at the peripheries of the anterior and middle cerebral arteries was maintained, and an Allcock test confirmed a fistula connecting from the C3 region of the internal carotid artery to the cavernous sinus. Transvenous target embolization of the fistula was performed while protecting the internal carotid artery using a balloon. The use of only a single coil led to the disappearance of the shunt.

Conclusion: In some patients, a small, direct CCF can be cured by target embolization. It is important to predict the size of a fistula and clearly visualize the fistulous site in the process of diagnosis.

Keywords $>$ direct carotid-cavernous fistula, target embolization, Allcock maneuver, transvenous approach

\section{Introduction}

Many direct carotid-cavernous fistulas are difficult to treat due to high flow. However, target coil embolization is possible in some patients with small fistula. In this study, we report on a patient with a non-traumatic direct carotidcavernous fistula (CCF) in whom the fistula site could be transvenously embolized using a single coil alone.

\section{Case Presentation}

A 74-year-old female was referred to our department with right pulsatile tinnitus, which had persisted for 2 months, and diplopia, which had persisted for 2 weeks. Slight right conjunctival congestion and right abducens nerve palsy

Department of Neurosurgery, Wakayama Rosai Hospital, Wakayama, Wakayama, Japan

Received: April 1, 2016; Accepted: August 2, 2016

Corresponding author: Nobuhide Hayashi. Department of Neurosurgery, Wakayama Rosai Hospital, 93-1 Kinomoto, Wakayama, Wakayama 640-8505, Japan

Email: nbh-hayashi@wakayamah.johas.go.jp

(C)2016 The Editorial Committee of Journal of Neuroendovascular

Therapy. All rights reserved. were noted. Vascular murmurs involving the right orbit to posterior ear auricle were heard. Magnetic resonance angiography (MRA) revealed a right cavernous sinus (CS) and right inferior petrosal sinus (IPS), suggesting a CCF. Cerebral angiography was performed.

\section{Cerebral angiography}

Angiography of the right internal carotid artery (ICA) showed a right $\mathrm{CS}$ in the arterial phase. At the peripheries of the anterior cerebral artery (ACA) and middle cerebral artery (MCA), anterograde blood flow was confirmed (Figs. 1A and 1B). The main venous outflow tract was the right IPS, but reflux to the Sylvian vein was partially observed (Fig. 1C). There was no visualization of the ipsilateral superior orbital vein (SOV), superior petrosal sinus (SPS), or contralateral CS. There was also no involvement of the external carotid artery system. According to Barrow's classification, a diagnosis of a type A direct CCF was made. ${ }^{1)}$ On a Matas test by angiography of the left ICA, there was no visualization of the fistula due to the deletion of the anterior communicating artery (A-com). However, on a lateral view of an Allcock test, the fistulous site was visualized through the posterior communicating artery (P-com). The fistula was present as a semilunar shunted 



Fig. 1 Diagnostic angiograms. (A) and (B) Anteroposterior (A) and lateral view (B) of the right carotid angiogram are shown. The cavernous sinus and right inferior petrosal sinus are opacified in the early arterial phase. The ipsilateral anterior and middle cerebral artery are fully illustrated. (C) Anteroposterior view of the right internal angiogram in the late arterial phase is shown. Retrograde leptomeningeal venous drainage into the superficial middle cerebral vein is illustrated (black arrows). (D) Lateral view of the Allcock test illustrates the fistulous site (black arrowhead).

pouch between the right ICA (C3 region) and former half of the CS (Fig. 1D).

\section{Treatment plan}

Endovascular treatment was indicated for the symptomatic, direct $\mathrm{CCF}$ with cortical venous reflux. On angiography of the right ICA, both the ipsilateral ACA and MCA were anterogradely visualized, suggesting that the fistulous size is small. We assumed that an access to the fistulous site visualized on the Allcock test might facilitate target embolization of the fistula. An original MRA image suggested the presence of a fistula at the junction between the dorsal side of the right ICA (C3 region) and ventromedial side of the right CS (Fig. 2). As a therapeutic strategy, the outflow tract to the Sylvian vein was initially embolized, and, subsequently, target coil embolization was performed through an access to the fistulous site. On the ICA side, a compliant balloon for embolizing aneurysms was used to prevent coil protrusion. On the arterial side, a guiding catheter with a balloon was used to prevent coil migration. Considering that an access from the venous side to the fistulous site may be impossible, a guiding catheter with a large inner diameter was prepared. For an Allcock test, a diagnostic catheter for the vertebral artery (VA) was prepared.

\section{Endovascular treatment}

Under general anesthesia, embolization was performed. A $9 \mathrm{Fr}$ sheath was inserted into the right femoral artery, a $4 \mathrm{Fr}$ sheath into the left femoral artery, and a $6 \mathrm{Fr}$ sheath into

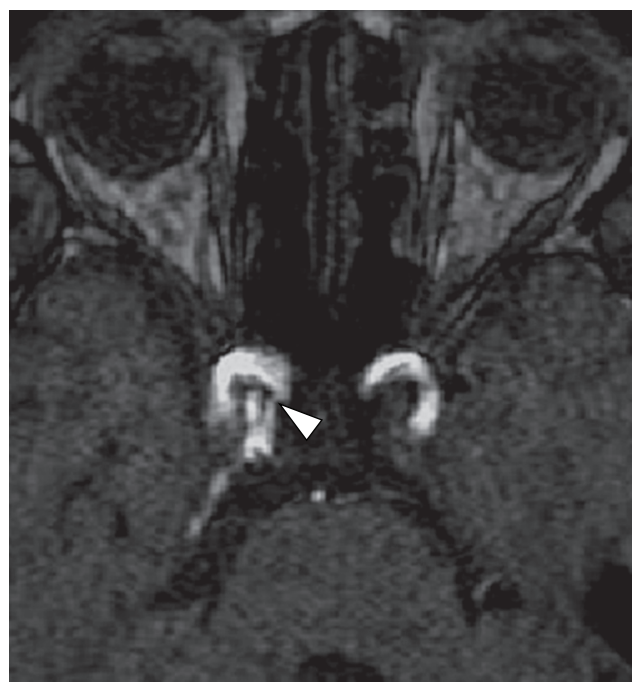

Fig. 2 A source image of MRA. The dorsal side of the right internal carotid artery and ventral side of the cavernous sinus are connected through a small fistula (white arrowhead).

the right femoral vein for systemic heparinization. A $9 \mathrm{Fr}$ Optimo (Tokai Medical Products, Aichi, Japan) was guided to the right ICA, a 4 Fr BHW (Katecs Co., Ltd., Osaka, Japan) to the right VA, a 6 Fr shuttle sheath (Cook Medical, Bloomington, IN, USA) to the right internal jugular vein, and a 6 Fr Envoy STR (Codman \& Shurtleff, Johnson \& Johnson, Raynham, MA, USA) coaxially to the right IPS. A CHIKAI $14(200 \mathrm{~cm})$ (Asahi Intec, Aichi, Japan) and SL-10 45 (Stryker, Kalamazoo, MI, USA) were guided into the CS through the right IPS, and the outflow tract to the 


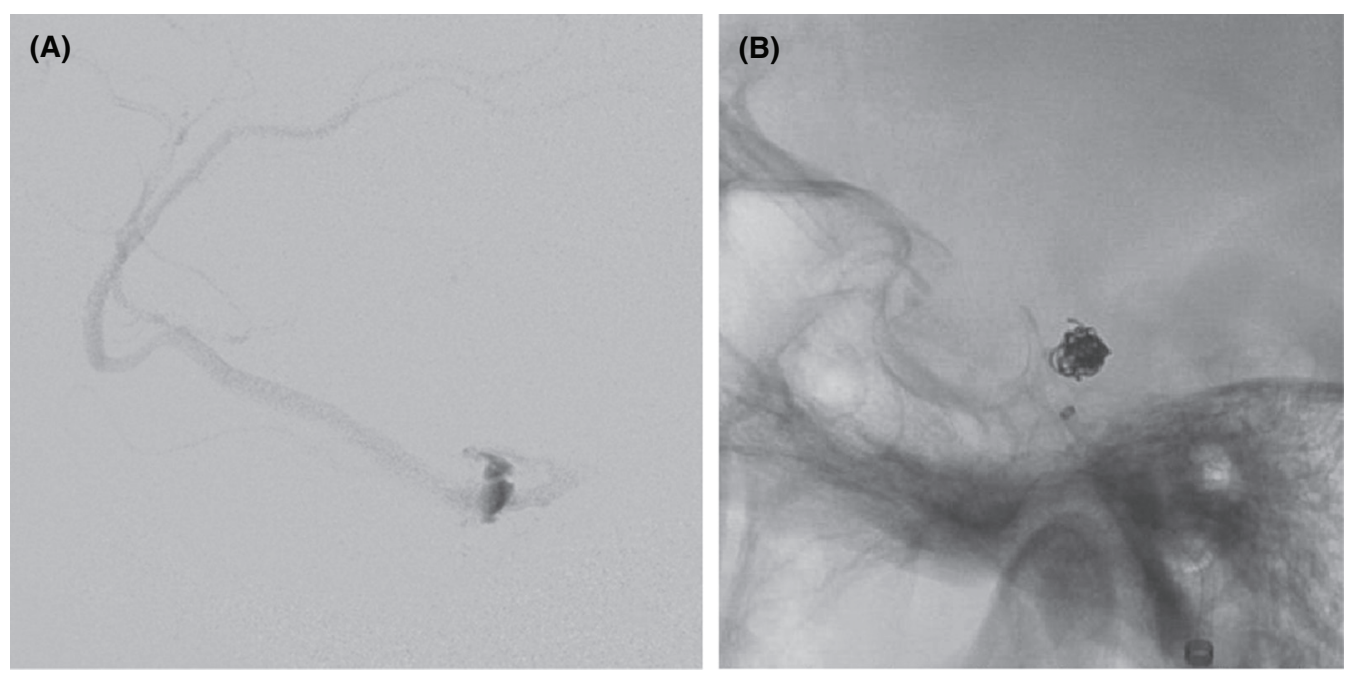

Fig. 3 (A) Cortical venous reflux into superficial middle cerebral vein is illustrated. (B) 3 coils (Axium ( $3 \mathrm{~mm} \times 8 \mathrm{~cm}$ ), Axium $(2.5 \mathrm{~mm} \times 6 \mathrm{~cm})$, ED coil $(2 \mathrm{~mm} \times 8 \mathrm{~cm}))$ are placed in the outlet of the cortical drainage.
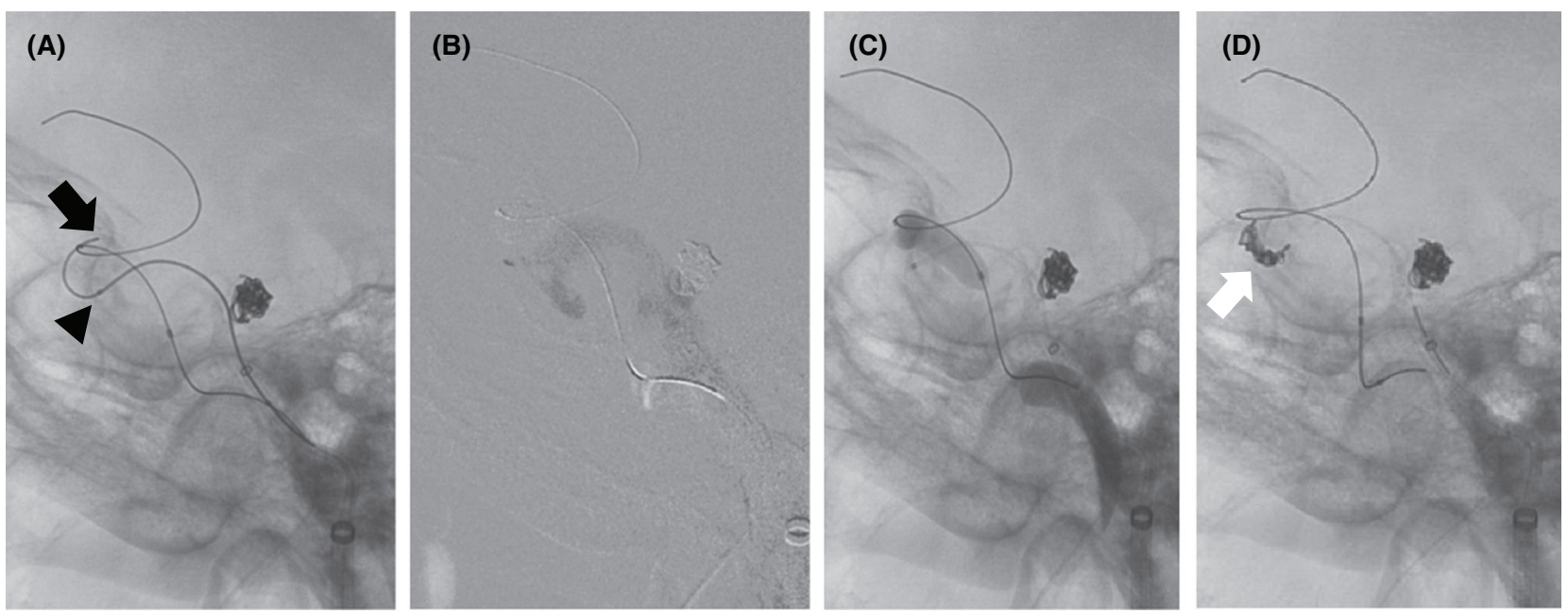

Fig. 4 Access to the fistulous point and embolization. (A) The tip of the microguidewire (black arrow) is advanced into the right carotid artery from the venous side through the fistula, and the tip of the microcatheter (black arrowhead) is placed just behind the fistulous site. (B) Whole image of the cavernous sinus is illustrated by microcatheter angiography. (C) HyperGlide $(4 \times 10 \mathrm{~mm})$ is inflated in order to prevent the coil from projecting into the internal carotid artery. (D) A Galaxy coil ( $3.5 \mathrm{~mm} \times 7.5 \mathrm{~cm}$, white arrow) is placed in the fistulous site.

Sylvian vein was embolized using three coils (Axium, $3 \mathrm{~mm}$ $\times 8 \mathrm{~cm},(\mathrm{eV} 3$ Covidien, Irvine, CA, USA), Axium, $2.5 \mathrm{~mm} \times$ $6 \mathrm{~cm}$ (eV3 Covidien, Irvine, CA, USA), ED coil, $2 \mathrm{~mm} \times$ $8 \mathrm{~cm}$ (KANEKA MEDIX CORP., Osaka)) (Figs. 3A and 3B). Subsequently, angiography of the right VA was performed using the Allcock maneuver by balloon occlusion of the right ICA to visualize the fistulous site. Under a road map, the fistula was investigated, and the CHIKAI 14 could be guided to the right ICA beyond the fistula. The SL-10 $45^{\circ}$ was guided to an area adjacent to the fistulous site so that it followed the CHIKAI 14 (Fig. 4A), and contrast-enhanced angiography through the SL- $1045^{\circ}$ confirmed that the entire area was visualized as an outflow tract (Fig. 4B). While inflating a HyperGlide $4 \times 10 \mathrm{~mm}$ (eV3 Covidien, Irvine, CA, USA) in the right ICA (C3-C4 regions) (Fig. 4C), a Galaxy $3.5 \mathrm{~mm} \times 7.5 \mathrm{~cm}$ (Codman \& Shurtleff, Johnson \& Johnson, Raynham, MA, USA) was used as the first coil. The coil was placed so that it occluded a fistula-connected semilunar shunted pouch without deviating into the ICA (Fig. 4D). Considering the risk of arterial blood flowrelated coil migration, two balloons (Optimo and HyperGlide) that had been inflated were deflated in the order of HyperGlide and Optimo to confirm the absence of coil migration. The two balloons were again inflated for coil removal, and deflated using similar procedures to confirm the absence of coil migration. Embolization with this coil 

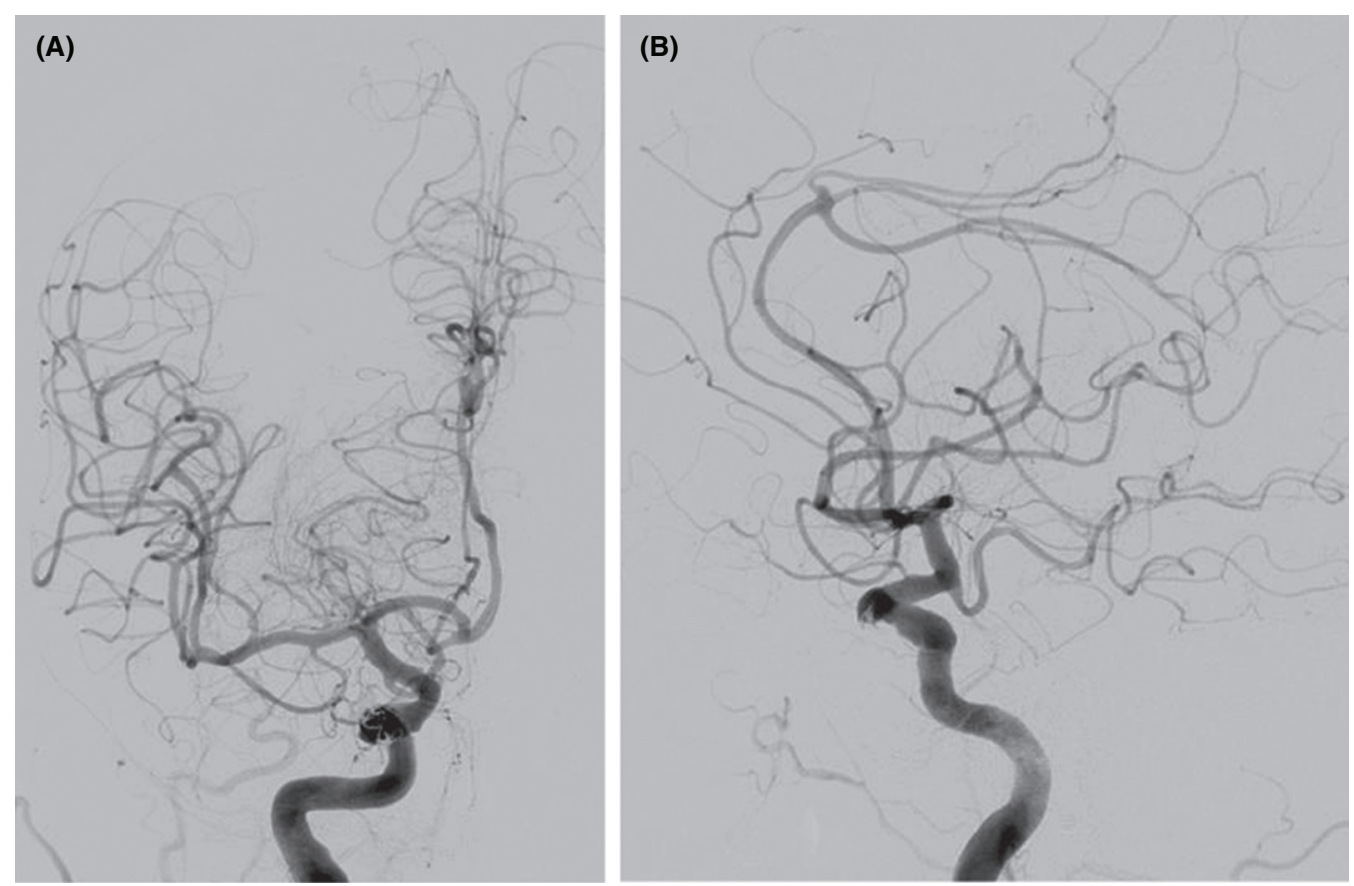

Fig. 5 Final angiograms. (A) and (B) Anteroposterior view (A) and lateral view (B) of the right carotid angiogram in the early arterial phase shows complete disappearance of the fistula.

alone led to the complete disappearance of shunt blood flow (Figs. 5A and 5B). Subsequently, clinical symptoms also gradually disappeared in a few days. Follow-up cerebral angiography after 6 months did not reveal recurrence. The course has been favorable in the absence of symptoms.

\section{Discussion}

Essential treatment for a direct $\mathrm{CCF}$ is closure of the fistulous site. Some patients respond to target embolization, depending on the size and shape of a fistula. In the present case, preoperative diagnostic angiography suggested a small fistula, and the fistulous site could be estimated on an Allcock test; therefore, treatment by target embolization was possible. As an embolization procedure, the fistula was tightly occluded using a single coil alone by adopting a compliant balloon on the arterial side using an approach through the venous side.

To predict the size of a fistula, diagnostic angiography findings are important. A study classified the fistulous size into three categories: i) small when both the ACA and MCA are visualized; ii) medium when only the MCA is visualized, and iii) large when neither the ACA nor MCA is visualized, and reported that small fistulas accounted for $12.8 \%{ }^{2)}$ Another study classified the fistulous size into three categories: i) small when a sufficient volume of blood flow at the periphery is confirmed on angiography of the internal carotid artery; ii) medium when there is no fistulous visualization through the contralateral ICA despite a decrease in peripheral blood flow or its disappearance, and iii) large when a fistula is visualized through the contralateral ICA or P-com in the absence of peripheral blood flow, and indicated that small fistulas accounted for $21.9 \% .^{3)}$ These classifications have a limitation: individual differences in A-com and P-com developments are not considered. However, they are useful for simply predicting the fistulous size.

The Allcock maneuver was useful for visualizing the fistulous site. The fistula was visualized as a narrow site connecting from the ICA ( $\mathrm{C} 3$ region) to the $\mathrm{CS}$ on a lateral view of an Allcock maneuver. If P-com development is insufficient, the fistulous size may be evaluated using the Matas maneuver. However, on the Allcock maneuver, the fistulous site may be more readily visualized, as there is no duplication of the contralateral ICA on a lateral view. If the Matas or Allcock maneuver is combined with 3D rotational angiography (3DRA), favorable working projection of the fistulous site may be maintained. ${ }^{4,5}$ When a fistula is large, it is difficult to visualize the fistulous site clearly, ${ }^{5,6)}$ but a study reported that the fistulous site could be visualized by performing manual exsanguination through the ICA on an Allcock maneuver or occluding the ICA using a guiding catheter with a balloon and slowly infusing a contrast medium to control the fistula contrast medium volume.6) 
In the present case, the three-dimensional position of the fistulous site was evaluated based on an original MRA image. However, if contrast-enhanced cone beam CT is performed using a flat-panel-type angiograph, a more detailed anatomical structure, involving the association with the bone structure, may be examined.

Coil embolization is effective for small, direct CCF. A study introduced coil embolization of slow-flow direct $\mathrm{CCF}$ through a transvenous approach. ${ }^{7)}$ However, in the literature, many studies reported the results of transarterial embolization. ${ }^{3,8-10)}$ According to a study, ${ }^{9)}$ transarterial coil embolization was performed for 12 patients with small, direct $\mathrm{CCF}$, and the mean number of coils was 8.4 (2-16). Another study presented five patients who underwent transarterial embolization with a balloon for the ICA, with a mean coil count of $11.2(6-18) .{ }^{11)}$ In these studies, the number of coils was within the permissible range, but it is difficult to stabilize a coil in the venous sinus using a transarterial approach; the number of coils tends to be large. If a microcatheter is guided from the venous side, the fistulous site may be embolized in a narrow extent by occluding the ICA side with a balloon to prepare a wall; therefore, in the present case, we selected a transvenous approach. The use of a balloon prevents coil deviation into the ICA, ischemic cerebral complications, ${ }^{12}$ and blood flow-related coil migration. On the other hand, a transarterial approach may be useful in cases in which a transvenous access to the fistulous site is difficult, those in which large fistulas make tight packing of the fistulous site using a transvenous approach alone difficult, and those in which it is difficult to guide a balloon to the ICA.

In many cases, it is difficult to treat large, direct CCF. Previously, detachable balloons were used, but the procedure is not technically simple. Early detachment-related complications and balloon rupture-related recurrence ${ }^{13)}$ occurred, leading to a failure rate of $5 \%$ to $10 \%{ }^{14)}$; currently, detachable balloons are not used. For coil embolization of large fistulas, sinus packing is required in some cases, and cranial nerve palsy may occur. ${ }^{15)}$ The usefulness of liquid embolizing substances, such as Etylene vinyl oxide copolymer (Onyx) (eV3 Covidien, Irvine, CA, USA) and N-butyl-2-cyanoacrylate (NBCA), has been reported, but glue migration-related complications may occur. ${ }^{16)}$ In some cases in which sinus packing is required, an access to the SOV or cortical outflow tract becomes difficult in the latter half of the procedure regardless of the type of embolizing substance; embolization of the venous outflow tract should be predominantly conducted. In the present case, embolization of the cortical venous outflow tract was initially performed, but it may not have been necessary based on the results of treatment. Furthermore, we used a guiding catheter with a 9 Fr balloon, considering an access from the arterial side. However, a 7 Fr balloon may have been sufficient even when using a single microcatheter on the arterial side in addition to a compliant balloon.

Etiological factors for non-traumatic direct $\mathrm{CCF}$ include ruptured cerebral aneurysms, Ehlers-Danlos syndrome, fibromuscular dysplasia, idiopathic, and iatrogenic (transnasal endoscopic surgery, cerebral endovascular treatment) factors. ${ }^{8}{ }^{17)}$ In the present case, there was no history of trauma or other concomitant disease, and angiography did not show cerebral aneurysm formation; therefore, a diagnosis of an idiopathic direct CCF was made. Although the etiology of idiopathic direct $\mathrm{CCF}$ remains to be clarified, a wall fragilityrelated fissure may lead to a direct $\mathrm{CCF} .{ }^{18)}$ In the present case, reflux to the cortical vein was observed, and right pulsatile tinnitus, right conjunctival congestion, and diplopia related to right abducens nerve palsy were noted; therefore, endovascular treatment was indicated. Although some indirect $\mathrm{CCF}$ spontaneously occlude in the absence of treatment, direct CCF do not spontaneously occlude due to high flow. ${ }^{8)}$ In the present case, inferior venous outflow through the right IPS was primarily observed, and cortical vein reflux was relatively slight. However, if long-term pressure loading results in the progression of thrombosis of the venous sinus, reflux to the SOV or cortical vein reflux may be enhanced, deteriorating ocular symptoms or inducing venous congestion or intracranial hemorrhage. According to a study regarding traumatic direct CCF, cortical vein reflux was noted in 20 (62.5\%) of 32 patients. ${ }^{4}$ The presence of cortical vein reflux may lead to intracranial hemorrhage, as indicated for dural arteriovenous fistula (dAVF).

Small, direct CCF may be cured by target coil embolization in some patients. The prediction of the fistulous size and visualization of the fistulous site are key points for direct CCF treatment. In the present case, the fistulous size could be predicted on diagnostic angiography, and it was possible to visualize the fistula on the Allcock maneuver, contributing to the successful results of target embolization. The use of a balloon on the arterial side and microcatheter guidance from the venous side facilitated fistulous closure with a small number of coils.

\section{Conclusion}

We reported on a patient with a non-traumatic direct $\mathrm{CCF}$ who underwent target embolization of the fistulous site with a 
single coil through a transvenous approach. It is important to evaluate the fistulous size and site before endovascular treatment. In the present case, the Allcock maneuver was useful.

\section{Disclosure Statement}

The first author and co-authors have no conflicts of interest.

\section{References}

1) Barrow DL, Spector RH, Braun IF, et al: Classification and treatment of spontaneous carotid-cavernous sinus fistulas. J Neurosurg 1985; 62: 248-256.

2) Chi CT, Nguyen D, Duc VT, et al: Direct traumatic carotid cavernous fistula: angiographic classification and treatment strategies. Study of 172 cases. Interv Neuroradiol 2014; 20: 461-475.

3) Malan J, Lefeuvre D, Mngomezulu V, et al: Angioarchitecture and treatment modalities in posttraumatic carotid cavernous fistulae. Interv Neuroradiol 2012; 18: 178-186.

4) Kohyama S, Ishihara S, Yamane F, et al: Three-dimensional digital subtraction angiography for endovascular treatment of direct carotid-cavernous fistula -case report-. Neurol Med Chir (Tokyo) 2010; 50: 404-406.

5) Nishio A, Kawakami T, Mitsuhashi Y, et al: Usefulness of intravascular ultrasonography monitoring of coil embolization for traumatic direct carotid-cavernous fistula-case report-. Neurol Med Chir (Tokyo) 2009; 49: 604-607.

6) Tsuji K, Nakagawa N, Fukawa N, et al: A novel technique for identifying the fistulous point in a direct carotidcavernous fistula. J Clin Neurosci 2016; 25: 152-155.

7) Su IC, Cruz JP, Krings T: Low-flow direct carotid-cavernous fistula caused by rupture of an intracavernous carotid aneurysm. Interv Neuroradiol 2014; 20: 476-481.

8) Aralasmak A, Karaali K, Cevikol C, et al: Venous drainage patterns in carotid cavernous fistulas ISRN Radiology 2014; Article ID 760267, 7 pages.
9) Chao-B L, Michael M-H T, Chung-J L, et al: Transarterial embolization of traumatic carotid-cavernous fistulae by gugliemi detachable coils. A seven-year experience. Interv Neuroradiol 2008; 14: 5-8.

10) Kobayashi N, Miyachi S, Negoro M, et al: Endovascular treatment strategy for direct carotid-cavernous fistulas resulting from rupture of intracavernous carotid aneurysms. AJNR Am J Neuroradiol 2003; 24: 1789-1796.

11) Andrade G, Ponte De Souza ML, Marques R, et al: Endovascular treatment of traumatic carotid cavernous fistula with balloon-assisted sinus coiling. A technical description and initial results. Interv Neuroradiol 2013; 19: 445-454.

12) De Renzis A, Nappini S, Consoli A, et al: Balloon-assisted coiling of the cavernous sinus to treat direct carotid cavernous fistula. A single center experience of 13 consecutive patients. Interv Neuroradiol 2013; 19: 344-352.

13) Hamid RS, Haq T-u, Shamim MS, et al: Endovascular approach as primary treatment for traumatic carotid cavernous fistula: local experience from Pakistan. J Pak Med Assoc 2011; 61: 989-993.

14) Naesens R, Mestdagh C, Breemersch M, et al: Direct carotidcavernous fistula: a case report and review of the literature. Bull Soc Belge Ophtalmol 2006; 43-54.

15) Nishino $\mathrm{K}$, Ito $\mathrm{Y}$, Hasegawa $\mathrm{H}$, et al: Cranial nerve palsy following transvenous embolization for a cavernous sinus dural arteriovenous fistula: association with the volume and location of detachable coils. J Neurosurg 2008; 109: 208-214.

16) Ramalingaiah AH, Prasad C, Sabharwal PS, et al: Transarterial treatment of direct carotico-cavernous fistulas with coils and Onyx. Neuroradiology 2013; 55: 1213-1220.

17) Mergeani A, Popescu D, Laza C, et al: A review on endovascular techniques for treatment of direct post-traumatic carotid-cavernous fistula supported by case presentations. Maedica (Buchar) 2012; 7: 332-338.

18) Ohta T, Matsutani M (Eds): Neurosurgery 10th edition. Kinpodo. Kyoto, 2008, 671. (in Japanese) 